\title{
Natimortalidade de fetos com peso de 2.500g no município do Rio de Janeiro: 2008-2017
}

\author{
Stillbirth of fetuses weighing 2,500g in the city of Rio de Janeiro: 2008-2017
}

Mortinato de fetos de $2.500 \mathrm{~g}$ en la ciudad de Río de Janeiro: 2008-2017

\begin{abstract}
RESUMO
Este estudo visa descrever aspectos assistenciais das mulheres que tiveram natimortos com peso maior ou igual a $2.500 \mathrm{~g}$ e que morreram antes ou durante os seus nascimentos, de mães residentes no município do Rio de Janeiro (MRJ) nos anos de 2008 a 2017. Trata-se de um estudo descritivo, retrospectivo, utilizando dados secundários, integrando variáveis relacionadas aos óbitos destes bebês, a partir dos dados secundários dos Sistemas de Informação sobre Mortalidade (SIM) e Nascidos Vivos (SINASC). A coleta dos dados ocorreu durante os meses de janeiro a março de 2019. A taxa de natimortalidade no período estudado foi de $10,1 / 1000$ nascidos e a taxa de natimortalidade de fetos com $2500 \mathrm{~g}$ ou mais foi de 2,42/1000 nascidos. A faixa etária materna mais frequente foi de 20 a 34 anos, a escolaridade de 4 a 11 anos de estudo, o parto vaginal foi a principal via de nascimento, o sexo masculino apresentou maior frequência de natimortalidade, a grande maioria dos óbitos foi antes do parto e tiveram como local de ocorrência, os hospitais. As afecções originadas no período perinatal foram as mais prevalentes e o declínio dos óbitos não investigados se deu em 2010, possível relação com a obrigatoriedade da vigilância destes óbitos a partir deste ano.
\end{abstract}

DESCRITORES: Complicações na Gravidez; Cuidado Pré-natal; Natimorto; Peso Fetal.

\section{ABSTRACT}

This study aims to describe care aspects of women who had stillbirths with a weight greater than or equal to 2,500 g and who died before or during their births, of mothers residing in the city of Rio de Janeiro (CRJ) in the years 2008 to 2017. This is from a descriptive, retrospective study, using secondary data, integrating variables related to the deaths of these babies, based on secondary data from the Mortality Information Systems (SIM) and Live Births (SINASC). Data collection took place during the months from January to March 2019. The stillbirth rate in the studied period was $10.1 / 1000$ births and the stillbirth rate of fetuses with $2500 \mathrm{~g}$ or more was 2.42 / 1000 born. The most frequent maternal age group was 20 to 34 years old, schooling from 4 to 11 years of study, vaginal delivery was the main route of birth, the male gender had a higher frequency of stillbirth, the vast majority of deaths occurred before the birth and had as their place of occurrence, hospitals. Diseases originating in the perinatal period were the most prevalent and the decline in uninvestigated deaths occurred in 2010, possibly related to the mandatory monitoring of these deaths from this year on.

DESCRIPTORS: Pregnancy Complications; Prenatal Care; Stillbirth; Fetal Weight.

\section{RESUMEN}

Este estudio tiene como objetivo describir aspectos asistenciales de mujeres que tuvieron mortinatos con un peso mayor o igual a 2.500 gy que fallecieron antes o durante el parto, de madres residentes en el municipio de Río de Janeiro (MRJ) en los años 2008 a 2017. a partir de un estudio descriptivo, retrospectivo, utilizando datos secundarios, integrando variables relacionadas con la muerte de estos bebés, a partir de datos secundarios de los Sistemas de Información de Mortalidad (SIM) y Nacidos Vivos (SINASC). La recolección de datos se llevó a cabo durante los meses de enero a marzo de 2019. La tasa de mortinatos en el período estudiado fue de 10.1 / 1000 nacimientos y la tasa de mortinatos de fetos con 2500 go más fue de 2,42 / 1000 nacidos. El grupo etario materno más frecuente fue de 20 a 34 años, escolaridad de 4 a 11 años de estudio, el parto vaginal fue la principal vía de parto, el género masculino tuvo una mayor frecuencia de mortinatos, la gran mayoría de las muertes ocurrieron antes de la nacimiento y tuvo como lugar de ocurrencia los hospitales. Las enfermedades originadas en el período perinatal fueron las más prevalentes y la disminución de muertes no investigadas ocurrió en 2010, posiblemente relacionada con el control obligatorio de estas muertes a partir de este año.

DESCRIPTORES: Complicaciones del Embarazo; Cuidado Prenatal; Nacido Muerto; Peso Fetal.

RECEBIDO EM: 30/05/2020 APROVADO EM: 31/05/2020 


\section{Geiza Martins Barros}

Enfermeira. Doutoranda em Ciências da Saúde.

ORCID: 0000-0002-1269-5845

\section{Marcos Augusto Bastos Dias}

Médico.

ORCID: 0000-0003-1386-7001

\section{Valeria Saraceni}

Médica. Doutora em Saúde Pública. ENSP - Fiocruz. ORCID: 0000-0001-7360-6490

\section{INTRODUÇÃO}

A natimortalidade se configura como um problema em todo o mundo, tendo seu registro iniciado em 1928 na Inglaterra e País de Gales. Já em países como o Brasil, se tornou objeto de registro mais tardiamente e, atualmente, ainda é pouco estudada, reflexo da baixa visibilidade para a sociedade, embora, grande parte seja de mortes preveníveis por ações dos serviços de saúde $e^{(1,2)}$.

Segundo estimativas, ocorrem no mundo cerca de 2 a 6 milhões de natimortos de terceiro trimestre por ano ${ }^{(2)}$. As características socioeconômicas estão associadas a estes óbitos, sendo os países de baixa e média rendas os que as possuem em maior frequên$\mathrm{cia}^{(2,3)}$. Estima-se que 98\% dos óbitos fetais ocorra em países em desenvolvimento ${ }^{(4)}$.

No Brasil, as regiões com maiores taxas de natimortalidade são a Norte e a Nordeste, segundo estudo publicado em 2017. Essas regiões possuem os piores índices econômicos e sociais do país. A análise do estudo em questão apontou como determinantes da natimortalidade: a região de moradia, idade materna (avançada e precoce); e como excludentes dos determinantes, a pobreza e a etnia ${ }^{(1)}$.

Um dado de grande relevância é que em sua maioria esses são óbitos evitáveis. A série do Lancet "Pelo Fim da Natimortalidade Evitável” afirma que metade dos óbitos anuais (1,3 milhões) ocorre durante o trabalho de parto e nascimento e possuem, em sua grande maioria, condições evitáveis. $\mathrm{Na}$ realidade brasileira, a evitabilidade está na resolução de problemas na atenção pré-natal, na captação das gestantes e na identificação precoce de risco gestacional ${ }^{(4-6)}$.
No Brasil, as regióes com maiores taxas de natimortalidade

são a Norte e a

Nordeste, segundo

estudo publicado em

2017. Essas regióes

possuem os piores

índices econômicos e

sociais do país.

A denominação da morte como evitável conta com diferentes classificações e, cada uma delas, com critérios específicos. Tem-se registro deste tipo de classificação no Chile em 1979, por Erica Taucher, médica cirurgiã, e em 1980, na Europa, a classificação de Wigglesworth, patologista pediátrico. No Brasil, o primeiro método de classificação do óbito infantil foi desenvolvido por Luis Patrício Ortiz, mais conhecido como lista da Fundação Sistema Estadual de Análise de Dados de São
Paulo (Fundação SEADE). Em 2007, elaboraram a mais recente classificação brasileira de óbitos infantis denominada Lista de Causas de Mortes Evitáveis por Intervenções no Âmbito do Sistema Único de Saúde - SUS ${ }^{(7)}$.

Existe uma lacuna entre as taxas elevadas de óbitos com potencial evitabilidade e tal assunto receber pouca atenção por parte das políticas públicas e dos estudos científicos, além disso, mesmo com a recente Portaria do MS n. ${ }^{\circ}$ 72, de 11 de janeiro de $2010^{(8)}$ que estabelece a obrigatoriedade da vigilância do óbito fetal no SUS, ainda não temos a real dimensão destes óbitos e sua repercussão para as mulheres e suas famílias ${ }^{(6)}$.

Os óbitos fetais refletem as condições de vida de uma população e a qualidade dos serviços de saúde prestados e, são classificados de acordo com a causa base. $\mathrm{O}$ natimorto é um resultado adverso da gravidez e desafios na determinação de sua incidência compreendem a: registro tardio da gravidez; pré-natal insuficiente para determinação das condições maternas; falta de tecnologias para realizar, por exemplo, autopsia; ausência de um sistema único para classificar as causas de natimortos ${ }^{(9,10)}$.

Uma pesquisa na Holanda, país de nível econômico mais elevado, constatou que $65 \%$ de todos os natimortos ocorreu por causas placentárias. Neste mesmo estudo, abordaram as causas de "outros locais da Rede Global" com menos recursos e, $46,6 \%$ destes óbitos teve como causas atribuíveis à asfixia fetal intrauterina. Os autores argumentam que estas diferenças podem ter relação com a falta de um padrão único de classificação destes óbitos e, tomam como exemplo, a mulher grávida 
com pré-eclampsia (PE) que evolui para um natimorto, onde, para eles, a PE pode causar alterações placentárias e, por conseguinte, asfixia fetal e, a avaliação feita para análise do óbito pode escolher para qual direção seguir - materna (PE) ou condições placentárias ${ }^{(10)}$.

A mais recente estratégia de enfrentamento da natimortalidade, em nível Mundial, é o plano de ação para todos os recém-nascidos lançado em 2014 na Assembleia Mundial da Saúde (MAS), que por mil nascidos vivos de 18,4 em 2015 tabilidade destes óbitos ${ }^{(11)}$.

No Brasil, os sistemas de informação sobre Mortalidade (SIM) e Nascidos Vivos (SINASC) foram criados pelo DAvisa a redução do número de natimortos para 12 em 2030, como estratégia de evi-

TASUS e, têm a finalidade de obtenção de dados sobre mortalidade e nascimentos no país, além de subsidiarem os indicadores demográficos e de saúde. O SIM possui lacunas no que se refere à cobertura $\mathrm{e}$ qualidade das informações que variam de acordo com as regiões do país ${ }^{(12)}$. A análise dos dados de natimortalidade de fetos com 2500g ou mais de peso ao nascer, teoricamente viáveis, pode informar sobre o comportamento das taxas ao longo do tempo, as principais causas relacionadas aos óbitos e orientar políticas públicas de enfrentamento deste problema.

Diante deste contexto, esta pesquisa visa descrever aspectos assistenciais das mulheres que tiveram natimortos com peso maior ou igual a $2.500 \mathrm{~g}$ que morreram antes ou durante os seus nascimentos,

\section{Gráfico 1. Taxa de óbito fetal. Rio de Janeiro, RJ, Brasil, 2008-2016}

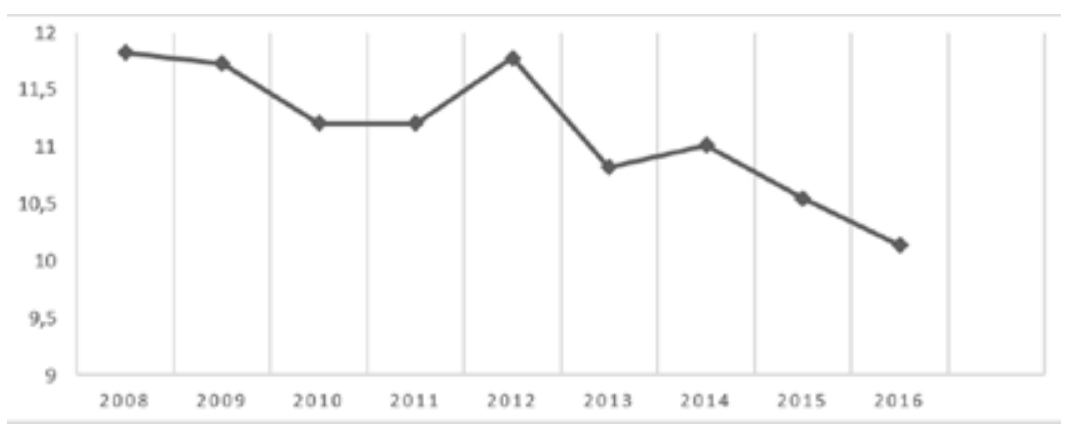

Gráfico 2. Números de natimortos com peso maior ou igual a 2.5000g e Capítulos da CID-10. Rio de Janeiro, RJ, Brasil, 2008-2016

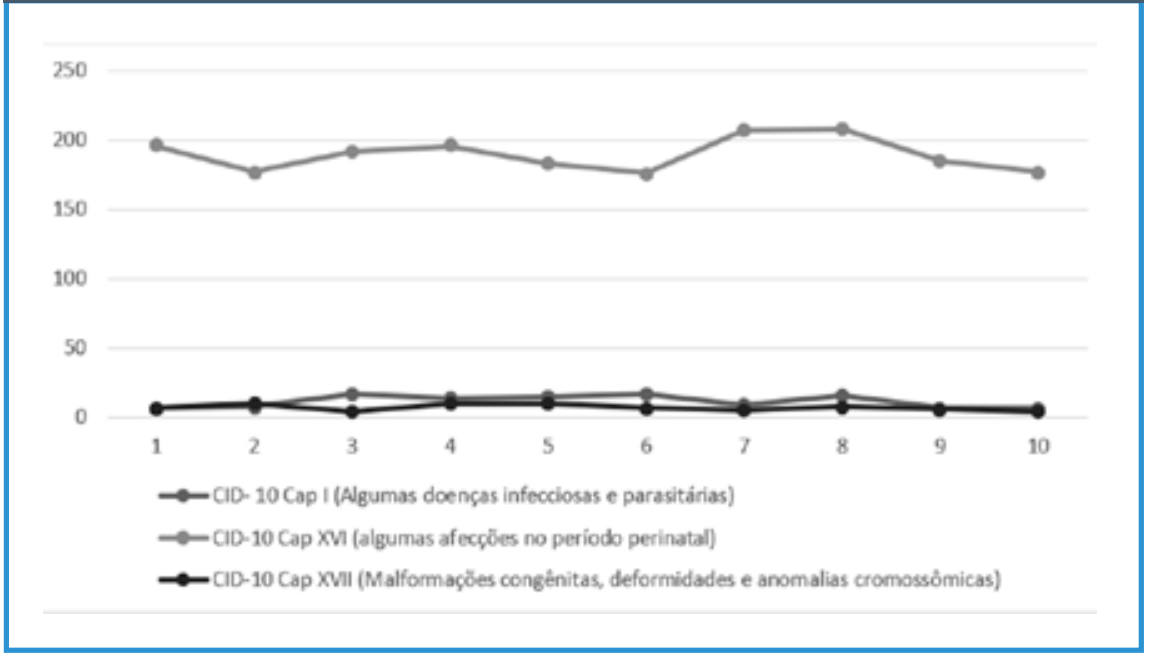

no município do Rio de Janeiro (MRJ) nos anos de 2008 a 2017.

\section{METODOLOGIA}

Estudo com abordagem quantitativa, retrospectivo e descritivo. Os últimos dez anos com dados disponíveis nos sistemas foram selecionados para a análise. A coleta dos dados se deu por meio do Departamento de Informática do Sistema Único de Saúde (DATASUS).

As variáveis utilizadas e disponíveis nos dados agregados do TABNET foram: óbito por residência/segundo município/ taxa de óbito fetal no MRJ, peso ao nascer, sexo, local de ocorrência, idade da mãe, escolaridade da mãe, tipo de parto, óbito relação ao parto, óbito investigado, capítulo CID10. Os critérios de busca foram: bebês com peso maior ou igual a $2.500 \mathrm{~g}$ que morreram antes ou durante os seus nascimentos, nos hospitais localizados no município do Rio de Janeiro (MRJ) nos anos de 2008 a 2017. A coleta dos dados ocorreu nos meses de janeiro, fevereiro e março de 2019. Os dados foram armazenados e analisados no programa Microsoft Excel 2010.

A taxa de mortalidade fetal em cada ano, foi calculada a partir do seguinte método: Número de Óbitos Fetais (22 semanas de gestação e mais), de mães residentes/ Número de nascimentos totais de mães residentes (nascidos vivos mais óbitos fetais de 22 semanas e mais de gestação) x $1000^{(8)}$.

\section{RESULTADOS}

\section{Aspectos assistenciais dos óbitos fetais com peso maior ou igual a $2.500 \mathrm{~g}$ segundo residência (MRJ) em uma década}

No período avaliado, ocorreram 2010 óbitos fetais com peso de nascimento maior ou igual a $2.500 \mathrm{~g}$, resultando em uma taxa média de 11,14 por 1000 nascimentos totais.

De acordo com o Gráfico 2 abaixo, o principal capítulo da CID-10 relacionado ao óbito fetal de peso maior ou igual a $2.500 \mathrm{~g}$, foi o XVI, que engloba afecções no período perinatal. 


\section{artigo}

Barros, G.M.; Dias, M.A.B.; Saraceni, V.;

Natimortalidade de fetos com peso de 2.500g no município do Rio de Janeiro: 2008-2017

Um importante instrumento de vigilância é a ficha de investigação dos óbitos fetais e esta tem boa aderência pelo MRJ, conforme demonstra o Gráfico 3.

$\mathrm{O}$ momento do óbito fetal em relação ao parto pode traduzir necessidade de aperfeiçoamento assistencial em serviços, como: pré-natal, emergência obstétrica e atenção ao parto. Quando este, ocorre, conforme Gráfico 4, maioria antes do parto, temos aspectos antecedentes a este como ponto de análise.

\section{DISCUSSÃO}

Em acordo com os dados apresentados no Gráfico 1, verificou-se que a taxa de mortalidade fetal apresentou leve flutua- ção ao longo dos dez anos, com tendência a declínio, exceto, nos anos de 2012 e 2014. A taxa de mortalidade fetal do MRJ está abaixo da meta estabelecida pela Assembleia Mundial da Saúde para 2030 $0^{(12,13)}$.

Em geral, os meninos morrem mais que as meninas em quase todas as causas e, de forma mais precoce ${ }^{(14)}$. E neste estudo, de todos os óbitos fetais com peso maior ou igual a $2.500 \mathrm{~g}$ nos anos analisados, o sexo masculino apresentou maior número de mortes. Em 2011 não houve registo do sexo de nascimento dos óbitos fetais no TABNET DATASUS ${ }^{(15)}$.

Um estudo realizado em 2012, também com dados do SIM referentes aos anos de 2000 a 2009, discutiu a vulnerabilidade do sexo masculino. Os natimortos do sexo
Gráfico 3. Número de natimortos e investigação dos óbitos. Rio de Janeiro, RJ, Brasil, 2008-2016

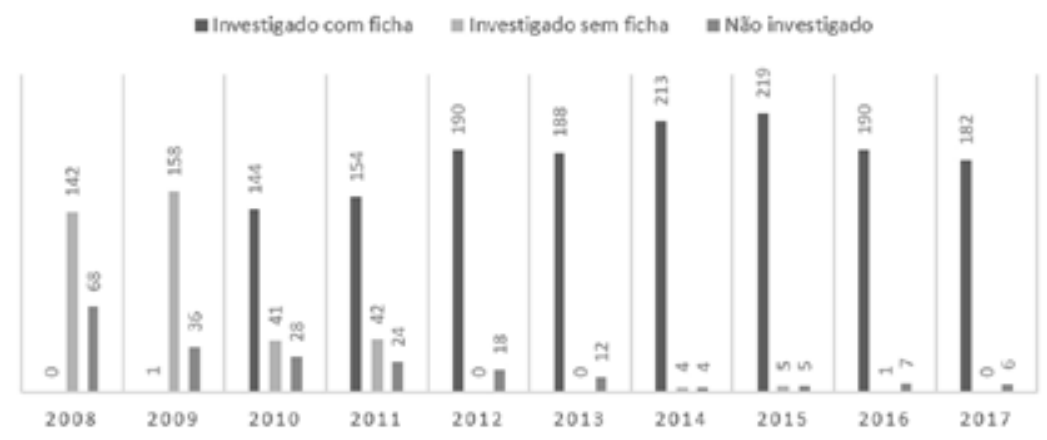

Gráfico 4. Momento do óbito em relação ao parto. Rio de Janeiro, RJ, Brasil, 2008-2016

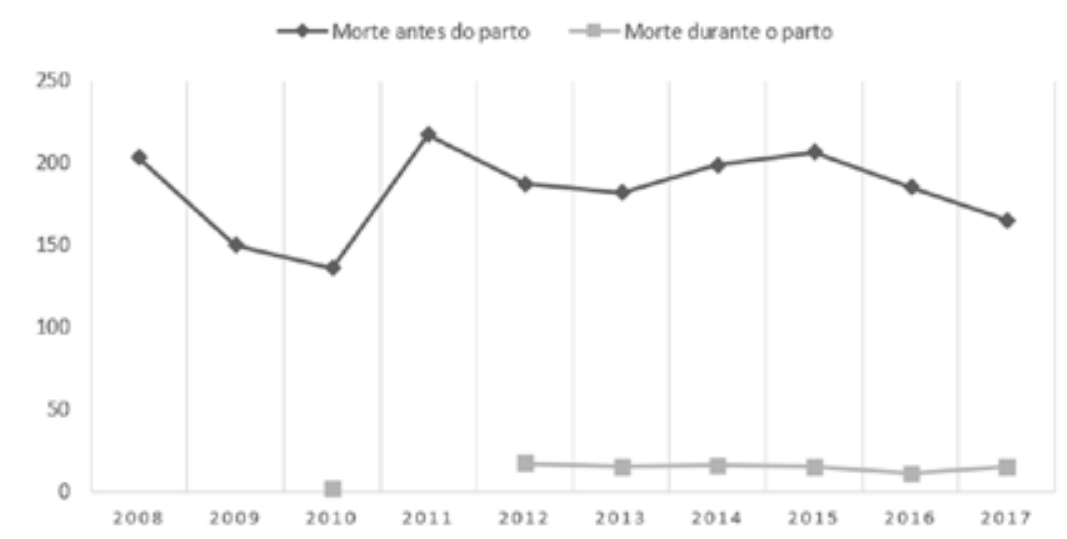

masculino foram mais frequentes que o feminino. Ainda segundo o estudo, as causas para os homens morrerem mais que as mulheres podem ser apontadas como relacionadas a fatores de risco e fatores relacionados às diferenças biológicas inatas, em que o masculino, estaria mais vulnerável aos efeitos imediatos de algumas doenças ${ }^{(16)}$.

$\mathrm{O}$ município apresentou um declínio dos óbitos não investigados, o qual se deu em 2010, possível relação com a obrigatoriedade da vigilância destes óbitos, a partir deste ano. A obrigatoriedade da vigilância do óbito fetal e infantil nos serviços de saúde (públicos e privados) que integram o SUS se deu através da Portaria n. ${ }^{\circ} 72$, de 11 de janeiro de $2010^{(8)}$.

No que se refere aos dados sociodemográficos, a idade da mãe mais prevalente foi de 20 a 34 anos e escolaridade de 4 a 11 anos de estudo. O que traduz um grupo de mulheres jovens e não integrantes do que se denomina "idade materna avançada" e, que pode aumentar a suspeita de que estas mortes sejam evitáveis. $\mathrm{O}$ conceito de morte evitável tem relação com a qualidade da atenção à saúde e pode ser compreendido como um "evento sentinela" (17).

Um importante dado constante no Gráfico 4 se refere à quantidade de bebês que morrem antes do parto. Cabe discutir que estes podem ter ido a óbito antes ou durante o trabalho de parto. $\mathrm{O}$ parto interpretado a partir dos seus períodos clínicos, temos que o primeiro período clínico abarca o que denominamos de trabalho de parto. Outra variável que poderia nos trazer importantes informações sobre essas mortes é o número de consultas de pré-natal e que não foi encontrado disponível nesta busca.

A via vaginal foi a de maior frequência nos nascimentos dos natimortos do estudo. Levando-se em conta que ao comparamos a via de nascimento no município do RJ de fetos vivos na mesma década, temos que em todos os anos, os bebês nasceram majoritariamente por cesarianas. Duas perguntas não conseguiremos responder apenas com este estudo: Mesmo sendo pequeno o número de natimortos no momento do parto em si, estes ocorreram antes ou durante o 
trabalho de parto? Quantos morreram durante a cesariana e parto vaginal?

Há grande quantidade de variáveis ignoradas. A escolaridade foi uma das mais ignoradas, o que pode sugerir inabilidade do profissional em reconhecê-la como relevante na causalidade do óbito fetal.

O ano de 2010 apresentou dados divergentes, pois apesar de apresentar as informações referentes aos óbitos fetais, registrou 75 óbitos pós-partos, o que caracterizam neomortos. Cabe ressaltar que todos os dados da tabela tiveram como base a inclusão destes, uma vez que não foi possível segregá-los. O número de ignorados (65) no quesito escolaridade foi outro dado que se destacou neste ano, pelo seu número maior em comparação aos demais anos.

De acordo com a Rede de Políticas Informadas por Evidências (EVIPnet), o óbito fetal compartilha causas com o óbito neonatal precoce (número de óbitos de $0 \mathrm{a}$ 6 dias de vida completos), reitera afirmando que ele é pouco conhecido no Brasil, mas que se insere nas ações para a redução da mortalidade materno-infantil. O óbito fetal integra a mortalidade perinatal, uma vez que esta inclui tanto os óbitos fetais quanto os neonatais precoces e, para ambos, têm-se causas preveníveis, como: ${ }^{(18)}$

1) Relacionadas à assistência: dificuldades no acesso e uso dos serviços de saúde, deficiente qualidade da assistência ao pré-natal, parto e recém-nascido;

2) Desigualdades sociais e econômicas;

3) Populações vulneráveis.

\section{CONCLUSÃO}

O óbito fetal com peso maior ou igual a $2.500 \mathrm{~g}$, peso este considerado ideal ao nascer, traz consigo questões relacionadas à saúde perinatal, qualidade da atenção e políticas públicas com foco em medidas preventivas. A maior parte dos óbitos no MRJ ocorreu por doenças relacionadas ao período perinatal e a via de parto não caracterizou risco adicional, visto que a maioria ocorreu antes do parto. Tal fato pode ter relação com a qualidade da atenção pré-natal e assistência em emergências obstétricas.

Estes óbitos causam grande impacto na vida das mulheres e familiares que vivenciaram a gravidez até o último trimestre e o desfecho negativo, muitas vezes, inesperado, tem forte potencial danoso. No MRJ, há registros de investigações destes óbitos conforme vimos, nos resta saber se os serviços recebem devolutivas e se estas são capazes de promover práticas preventivas a fim de reverter essa tragédia. -

\section{REFERÊNCIAS}

1. Carvalho TS, Pellanda LC, Dayle P. Stillbirth prevalence in Brasil: exploration of regional differences. J. Pediatr (Rio J). 2018; 94(2):200-206.

2. Camargo ABM. A natimortalidade e a mortalidade perinatal em São Paulo. São Paulo em Perspectiva. 2008 jan./jun.; 22(1):30-47.

3. Madhi AS, et al. Causes of stillbirths among women from South Africa: a prospective, observacional Studi. Lancet. 2019; 7.

4. Holanda AAS. Caracterização da mortalidade fetal em Pernambuco, de 2000 a 2011: casas e fatores associados. Fundação Oswaldo Cruz, Rio de Janeiro, 2013.

5. Fonseca SC, Coutinho ESF. Fatores de Risco para Mortalidade Fetal em uma Maternidade do Sistema Único de Saúde, Rio de Janeiro, Brasil: Estudo Caso-Controle. Cad. Saúde Pública. 2010 fev.; 26(2):240-252.

6. Melo CM, et al. Vigilância do óbito como indicador da qualidade da atenção à saúde da mulher e da criança. Ciência \& Saúde Coletiva. 2017; 12(10):3457-3465.

7. Dias BAS, et al. Classificações de evitabilidade dos óbitos infantis: diferentes métodos, diferentes repercussões? Cad. Saúde Pública. 2017; 33(5):e0012591.

8. Ministério da Saúde (BR). Portaria n. 72, de 11 de janeiro de 2010. Estabelece que a vigilância do óbito infantil e fetal é obrigatória nos serviços de saúde (públicos e privados) que integram o Sistema Único de Saúde (SUS). Brasilia (DF): MS, 2010.

9. Campos DA. Identificação das causas evitáveis, que levaram à mortalidade fetal e de crianças menores de um ano no Município de Angelândia-MG em 2013. Polo Teófilo Otoni. Minas Gerais. 2014.
10. Mc Cleare EM, Goldenberg R. Understanding causes of stillbirth moving in tb é right direction. Lancet Glob Health. 2019 Apr.; 7(4):e400-e401.

11. Barros OS, et al. Mortalidade fetal e os desafios para a atenção à saúde da mulher no Brasil. Rev Saude Publica. 2019; 53:12.

12. Queiroz BL, et al. Estimativas do grau de cobertura e da mortalidade adulta (45q15) para as unidades da federação no Brasil entre 1980 e 2010 Rev BRas epidemiol. 2017 mai.; 20(Suppl1):21-33.

13. Coordinator, Every Women Every Child. Cada Mulher, Cada Criança. Estratégia Global para a Saúde das Mulheres, das Crianças e dos Adolescentes (2016-2030). Nova York: EWEC; 2015.

14. Gomes R, Nascimento EF. A produção do conhecimento da saúde pública sobre a relação homem-saúde: uma revisão bibliográfica. Cad Saúde Pública. 2006 mai.; 22(5):901-11.

15. Ministério da Saúde (BR). Informações em Saúde. TABNET DATASUS. Disponível em: http://tabnet.datasus.gov.br/cgi/menu_ tabnet_php.htm

16. Filho ADPC, Laurenti R. O Sexo Masculino Vulnerável: razão de masculinidade entre os óbitos fetais masculinos. Cad. Saúde Pública. 2012 abr.; 28(4):720-728.

17. Malta DC, et al. Lista de causas de mortes evitáveis por intervenções do sistema único de saúde do Brasil, 2007. Epidemiol. Serv. Saúde. 2007 out.-dez.; 16(4):233-244.

18. Ministério da Saúde (BR). Relatório Anual Rede para Políticas Informadas por Evidências (EVIPNet Brasil) janeiro-dezembro de 2016. Brasilia (DF): MS, 2016. 